\title{
Approximation Simulation of Dynamics of Lattice Model for Comb Polymer Chains ${ }^{\dagger}$
}

\author{
Linxi ZHANG, * Jianmin XU, and Longshao ZHAO \\ Department of Physics, Hangzhou University, Hangzhou, \\ 310028, People's Republic of China
}

(Received January 12, 1994)

\begin{abstract}
We present an approximation simulation study on the dynamics of a comb polymer chain, such as $(-\mathrm{A}-\mathrm{BR})_{-}$, with and without excluded volume, where $\mathrm{R}$ is the side chain. The dynamics of comb polymer chains are studied by investigating the dynamics of the equifinal linear polymer chains, $\left(\mathbf{A}-\mathbf{B}^{\prime}\right)_{x}$, with the probability of movement of $\mathbf{B}^{\prime}$ bead $p$. It is found that relaxation times of the first three normal modes obey the relation $T_{k}(p)-(N-1)^{1.96} / k^{1.97} p^{0.70}$ in the absence of excluded volume, and $T_{k}(p)-T_{k l} p^{-\beta_{k}}\left(\beta_{1}=0.5, \beta_{2}=0.75, \beta_{3}=0.72\right)$ in the presence of excluded volume, where $N=2 x$ and $T_{k l}$ is the relaxation time of the first three normal modes $T_{k}$ of linear polymer chains $(k=1,2$, and 3$)$.

KEY WORDS Monte Carlo Simulation / Probability of Movement / Relaxation Time / Comb Polymer Chain /
\end{abstract}

A lattice model has been used to study of the behavior of polymer chains in solution. This technique has been also applied to study the dynamics of polymer chains in solution. The study of the relaxation of lattice models of polymer chains using Monte Carlo simulation was initiated by Verdier and Stockmayer. ${ }^{1}$ The original work was on isolated simple cubic (SC) lattice chains, and it was confirmed that, in the absence of excluded volume, the model shows essentially Rouse-like ${ }^{2}$ behavior in chain length, $N$, and mode number, $k$, dependence on the relaxation time. In the presence of excluded volume, a deviation from Rouse-like behavior is seen in both $N$ and $k$ dependence. Later, a tetrahedral lattice model, ${ }^{3}$ a bodycentred cubic (BCC), ${ }^{4}$ and a face-centred cubic (FCC) were developed to study the dynamics of polymer chains. We notice these studies focused on the dynamics of linear polymer chains. It is more important to study the dynamics of comb polymers or polymer chains with side chains because most polymer chains are included in such branched polymer chains. However, investigation of the dynamics of comb polymer is more difficult because of the complexity of the polymer chain structure. In this paper, approximation simulations are performed, and the dynamics of comb polymer chains, such as $(\mathrm{A}-\mathrm{BR})_{x}$, where $\mathrm{R}$ is the side chain, are studied. As effects of the side chain, bead B may move slower than bead A. Here the probability of movement of the bead $B$ should be introduced. Therefore, the dynamics of comb polymer chains, $(\mathrm{A}-\mathrm{BR}-)_{x}$, are approximately studied by simulation of linear polymer chains, $\left(-A-B^{\prime}\right)_{x}$, with the probability of movement of the bead $\mathrm{B}^{\prime} p$ $(1.0 \geq p>0.0)$ (see Figure 1). If the side chain $\mathrm{R}$ includes a large number of beads, bead $\mathrm{B}$ move slowly, and the probability $p$ is small. The side chain $\mathrm{R}$ may also affect the mobility

+ The project supported by National Natural Science Foundation of China.

* To whom correspondence should be addressed. 

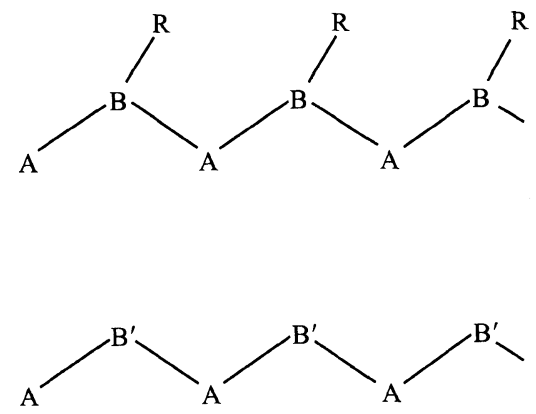

Figure 1. Equifinal linear polymer chain. (a) comb polymer chain $(\mathrm{A}-\mathrm{BR})_{x}$; (b) equifinal linear polymer chain $\left(-A-B^{\prime}\right)_{x}$, with probability of movement of $\mathbf{B}^{\prime}$ bead as $p$.

of bead A or mobility of the whole chain, or the effect is weak. This means that the dynamics of the equifinal linear polymer chains are different from linear polymer chains, and the probability of movement of $A$ bead, or the whole chain should be considered. The effects of $\mathrm{R}$ on $\mathrm{A}$ bead or the whole will be discussed in the future. In this paper, the dynamics are studied with calculation of relaxation times of the end-to-end vector $T_{\mathrm{R}}$ and relaxation times of the first three normal modes $T_{k}{ }^{1-5}$ The body-centred cubic (BCC) model is used here.

\section{MODEL}

The equifinal linear polymer chain is represented as a random walk of $N-1$ steps of unit length on an eight-choice body-centred cubic lattice $(N=2 x)$. Each step is referred to as a bond. The chain occupies $N$ lattice junction points. Each point is called a bead. The initial description refers to the case when excluded volume is present.

First a bead is chosen at random. If it is a $\mathbf{B}^{\prime}$ bead, as the $\mathbf{B}^{\prime}$ bead moves slower than the A bead, the probability of movement $p$ should be considered. If motion is not made, the bead cycle terminates. If motion is made, the chain moves according to the following algorithm.

If it is an end bead, there are seven possible motions corresponding to the seven possible location lying one lattice unit from the next to the last bead. One of these positions is chosen at random. If the new position is not occupied, the end bead moves and the cycle terminates. If the new position has been occupied, the end bead remains at its original position and the cycle terminates.

If an interior bead is chosen, conformation of the two adjacent beads is determined. This method has been described in detail previously. ${ }^{6}$

From time to time, chain conformation is sampled and the end-to-end vector $\boldsymbol{R}(t)$ and position of the $j$-th bead with respect to the origin $\boldsymbol{R}_{j}(t)$ are stored as functions of the number of bead cycles completed. The elementary time unit is taken as $N$ bead cycles.

Following Verdier and Kranbuel, the data are analyzed in terms of the end-to-end vector autocorrelation function defined as

$$
\rho_{\mathbf{R}}(t)=\langle\boldsymbol{R}(t) \cdot \boldsymbol{R}(0)\rangle /\left\langle R^{2}\right\rangle
$$

where $\boldsymbol{R}(t)$ is the end-to-end vector at a time $t$ ( $t$ in $N$ bead cycles) and $\langle>$ represent average approximated as a time average. The relaxation time, $T_{\mathrm{R}}$, is estimated by fitting an unweighted least-square line to the linear long-time region of a semilog plot of $\rho_{\mathrm{R}}(t) v \mathrm{~s}$. time. The inverse of the relaxation time is the negative of the slope of the line.

To analyze the dynamics in more detail, we also studied the relaxation of the first three normal modes. The normal modes $\boldsymbol{U}_{k}(t)$ are given by the Rouse formula

$$
\begin{aligned}
\boldsymbol{U}_{k}(t)= & \sum_{j=1}^{N}\left(\left(2-\delta_{k 0}\right) / N\right)^{1 / 2} \\
& \times \cos \left(\left(j-\frac{1}{2}\right) \pi k / N\right) \boldsymbol{R}_{j}(t)
\end{aligned}
$$

where $\boldsymbol{R}_{j}(t)$ is the position of the $j$-th bead with respect to the origin. The autocorrelation function of the $k$-th normal mode, $\rho_{k}(t)$, is given by

$$
\rho_{k}(t)=\left\langle\boldsymbol{U}_{k}(t) \cdot \boldsymbol{U}_{\mathbf{k}}(0)\right\rangle /\left\langle U^{2}\right\rangle
$$

The equilibrium average is again computed as 
a time average. The relaxation time of the $k$-th mode is compute by fitting a least-square line to a semilog plot of $\rho_{k}(t) v s$. time. The negative of the relaxation time is the inverse of slope of this line.

\section{RESULTS AND DISCUSSION}

Simulation performed on chains of length $12,24,36,48$, and 60 beads with the probability of movement of the $\mathbf{B}^{\prime}$ bead $p$ as $1.0,(0.75)$, $0.5,0.4,0.3,0.2$, and 0.1 with and without

Table I. Relaxation times, $T_{\mathrm{R}}$, as a function of chain length, $N$, and probability, $p$, for non-excluded volume and excluded volume

\begin{tabular}{|c|c|c|c|c|c|c|c|c|c|c|}
\hline \multirow{3}{*}{$p$} & \multicolumn{10}{|c|}{$T_{\mathbf{R}}$} \\
\hline & \multicolumn{5}{|c|}{ Non-excluded volume } & \multicolumn{5}{|c|}{ Excluded volume } \\
\hline & 12 & 24 & 36 & 48 & 60 & 12 & 24 & 36 & 48 & 60 \\
\hline 1.0 & 18.2 & 76.5 & 176 & 309 & 495 & 52.2 & 289 & 776 & 1460 & 2330 \\
\hline 0.75 & 21.7 & 93.0 & 207 & 355 & 571 & & & & & \\
\hline 0.5 & 26.9 & 133 & 279 & 482 & 820 & 68.0 & 333 & 851 & 1559 & 2590 \\
\hline 0.4 & 32.3 & 140 & 308 & 518 & 881 & 79.0 & 440 & 1250 & 2280 & 3561 \\
\hline 0.3 & 45.6 & 166 & 400 & 692 & 1140 & 101 & 541 & 1611 & 2926 & 4939 \\
\hline 0.2 & 55.6 & 224 & 520 & 891 & 1510 & 143 & 689 & 1961 & 3910 & 6069 \\
\hline 0.1 & 83.0 & 375 & 859 & 1530 & 2516 & 210 & 996 & 2721 & 5199 & 8269 \\
\hline
\end{tabular}

Table II. Relaxation times, $T_{k}$, as a function of chain length, $N$, mode number, $K$, and probability, $p$, for non-excluded volume ${ }^{a}$

\begin{tabular}{|c|c|c|c|c|c|c|c|c|c|c|c|}
\hline \multirow{2}{*}{$p$} & \multirow{2}{*}{$k$} & \multicolumn{2}{|c|}{$N=12$} & \multicolumn{2}{|c|}{$N=24$} & \multicolumn{2}{|c|}{$N=36$} & \multicolumn{2}{|c|}{$N=48$} & \multicolumn{2}{|c|}{$N=60$} \\
\hline & & a & b & a & $\mathrm{b}$ & a & $\mathrm{b}$ & $\mathrm{a}$ & $\mathrm{b}$ & $\mathrm{a}$ & b \\
\hline \multirow[t]{3}{*}{1.0} & 1 & 18.3 & 18.1 & 76.5 & 77.0 & 175 & 175 & 309 & 312 & 495 & 488 \\
\hline & 2 & 4.7 & 4.7 & 20.1 & 19.7 & 45.0 & 44.7 & 78.8 & 79.6 & 126 & 125 \\
\hline & 3 & 2.1 & 2.1 & 8.5 & 8.8 & 20.1 & 20.1 & 37.5 & 35.8 & 55.6 & 56.0 \\
\hline \multirow[t]{3}{*}{0.75} & 1 & 21.5 & 22.1 & 92.9 & 94.2 & 200 & 214 & 355 & 382 & 571 & 597 \\
\hline & 2 & 6.0 & 6.7 & 24.5 & 24.0 & 54.6 & 54.6 & 95.2 & 95.4 & 150 & 152 \\
\hline & 3 & 2.6 & 2.5 & 10.2 & 10.0 & 23.8 & 24.6 & 43.9 & 43.0 & 68.8 & 68.5 \\
\hline \multirow[t]{3}{*}{0.5} & 1 & 28.6 & 29.4 & 125 & 125 & 286 & 284 & 484 & 507 & 818 & 793 \\
\hline & 2 & 7.3 & 7.5 & 30.3 & 31.9 & 70.8 & 72.6 & 120 & 129 & 201 & 202 \\
\hline & 3 & 3.5 & 3.4 & 13.0 & 14.3 & 30.3 & 32.6 & 57.7 & 58.2 & 85.7 & 91.0 \\
\hline \multirow[t]{3}{*}{0.4} & 1 & 32.3 & 34.4 & 135 & 146 & 308 & 332 & 519 & 592 & 881 & 927 \\
\hline & 2 & 9.3 & 8.8 & 36.4 & 37.3 & 83.3 & 84.8 & 145 & 151 & 239 & 237 \\
\hline & 3 & 4.0 & 3.9 & 16.2 & 16.8 & 37.2 & 38.2 & 70.6 & 68.0 & 107 & 106 \\
\hline \multirow[t]{3}{*}{0.3} & 1 & 45.5 & 42.0 & 167 & 179 & 400 & 407 & 706 & 725 & 1143 & 1134 \\
\hline & 2 & 11.3 & 10.7 & 45.8 & 45.7 & 103 & 104 & 188 & 185 & 280 & 289 \\
\hline & 3 & 4.9 & 4.8 & 20.0 & 20.5 & 48.2 & 46.7 & 86.6 & 83.2 & 136 & 130 \\
\hline \multirow[t]{3}{*}{0.2} & 1 & 55.6 & 55.8 & 224 & 238 & 534 & 540 & 911 & 963 & 1500 & 1505 \\
\hline & 2 & 12.8 & 14.3 & 67.8 & 60.6 & 121 & 137 & 205 & 246 & 338 & 383 \\
\hline & 3 & 6.6 & 6.4 & 26.3 & 27.3 & 62.5 & 62.0 & 114 & 110 & 171 & 173 \\
\hline \multirow[t]{3}{*}{0.1} & 1 & 83.3 & 90.7 & 375 & 386 & 857 & 877 & 1533 & 1563 & 2500 & 2445 \\
\hline & 2 & 26.3 & 23.1 & 110 & 98.5 & 240 & 224 & 427 & 399 & 680 & 624 \\
\hline & 3 & 10.1 & 10.4 & 46.6 & 44.3 & 109 & 101 & 206 & 179 & 307 & 281 \\
\hline
\end{tabular}

a a, Monte Carlo simulation; b, values of quantities $0.165(N-1)^{1.96} p^{-0.7} k^{-1.97}$. 
excluded volume. All computations were performed on VAX8350 computer using FORTRAN source codes. At least 2000 runs were made for each case. The simulation requires approximately $9300 \mathrm{~min}$ of CPU time. Typical semilog plots of the end-to-end vector autocorrelation function $\rho_{\mathrm{R}}(t)$ for $N=60$ and probability of movement $p=0.5,0.3$, and 0.1

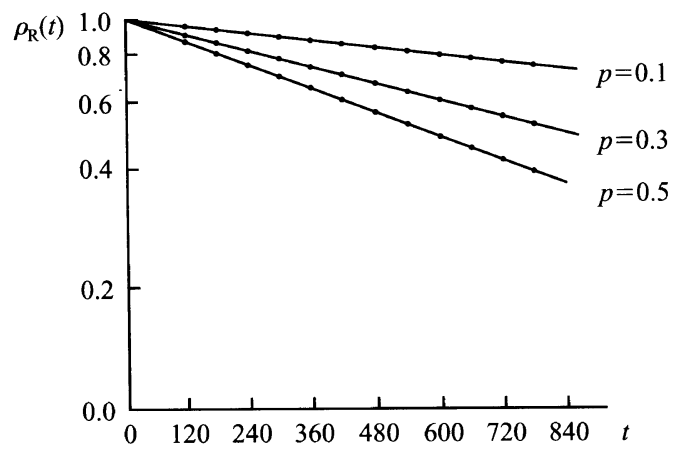

Figure 2. Semilogarithmic plots of the end-to-end vector autocorrelation function $\rho_{\mathrm{R}}(t) v s$. $t$ for various $p$ for chain length of $N=60$ in the absence of excluded volume. without excluded volume are shown in Figure 2. Autocorrelation functions for all other cases behave similarly. The decay appears to be linear (on the semilog plot) for long times. This indicates that the decay is exponential at long time. The relaxation times, $T_{\mathrm{R}}$, calculated from the long-time slopes of the $\ln \rho_{\mathrm{R}}(t) v s . t$ plots, are collected in Table I. For $p=1.0$, this means the side chain $\mathrm{R}$ is neglected, and the relaxation times, $T_{\mathrm{R} 1}$, obey the relation

$$
T_{\mathrm{Rl}}-(N-1)^{\alpha_{\mathrm{R}}}
$$

(relation between $N$ and $x$ is $N=2 x$ ). The values of $\alpha_{\mathrm{R}}$ in the absence and presence of excluded volume are 1.96 and 2.27 , respectively, which are close to the scaling predictions of 2.0 and 2.2. Our results are in good agreement with previous data. ${ }^{4,5}$ As effects of the side $R$, the relaxation time increases with decrease of $p$. In fact, the more beads in the side chain, the more slowly does B bead move, and the larger is the relaxation time.

Table III. Relaxation times, $T_{\mathbf{k}}$, as a function of chain length, $N$, mode number, $k$, and probability, $p$, for excluded volume ${ }^{a}$

\begin{tabular}{|c|c|c|c|c|c|c|c|c|c|c|c|}
\hline \multirow{2}{*}{$p$} & \multirow{2}{*}{$k$} & \multicolumn{2}{|c|}{$N=12$} & \multicolumn{2}{|c|}{$N=24$} & \multicolumn{2}{|c|}{$N=36$} & \multicolumn{2}{|c|}{$N=48$} & \multicolumn{2}{|c|}{$N=60$} \\
\hline & & $\mathrm{a}$ & b & $\mathrm{a}$ & $b$ & $\mathrm{a}$ & b & $\mathrm{a}$ & $\mathrm{b}$ & $\mathrm{a}$ & $b$ \\
\hline \multirow[t]{3}{*}{1.0} & 1 & 52.2 & & 293 & & 781 & & 1460 & & 2350 & \\
\hline & 2 & 10.7 & & 66.8 & & 168 & & 316 & & 523 & \\
\hline & 3 & 4.2 & & 27.2 & & 68.3 & & 139 & & 228 & \\
\hline \multirow[t]{3}{*}{0.5} & 1 & 69.9 & 76.7 & 331 & 431 & 856 & 1148 & 1580 & 2146 & 2580 & 3431 \\
\hline & 2 & 18.8 & 18.0 & 115 & 112 & 283 & 281 & 531 & 531 & 859 & 879 \\
\hline & 3 & 7.3 & 6.9 & 44.3 & 44.8 & 111 & 112 & 227 & 229 & 369 & 376 \\
\hline \multirow[t]{3}{*}{0.4} & 1 & 79.4 & 87.2 & 445 & 489 & 1249 & 1304 & 2281 & 2438 & 3549 & 3921 \\
\hline & 2 & 20.1 & 21.3 & 129 & 133 & 317 & 332 & 651 & 629 & 1051 & 1041 \\
\hline & 3 & 9.6 & 8.7 & 52.8 & 52.5 & 133 & 132 & 267 & 268 & 431 & 440 \\
\hline \multirow[t]{3}{*}{0.3} & 1 & 100 & 102 & 542 & 574 & 1532 & 1531 & 2920 & 2868 & 4935 & 4632 \\
\hline & 2 & 28.9 & 26.4 & 179 & 165 & 444 & 412 & 883 & 812 & 1462 & 1299 \\
\hline & 3 & 9.6 & 9.9 & 58.8 & 64.7 & 150 & 161 & 303 & 321 & 511 & 534 \\
\hline \multirow[t]{3}{*}{0.2} & 1 & 140 & 128 & 689 & 712 & 1951 & 1921 & 3889 & 3592 & 6063 & 5783 \\
\hline & 2 & 33.9 & 35.7 & 210 & 223 & 529 & 552 & 999 & 1043 & 1688 & 1747 \\
\hline & 3 & 14.2 & 13.4 & 88.9 & 86.8 & 253 & 218 & 493 & 449 & 865 & 727 \\
\hline \multirow[t]{3}{*}{0.1} & 1 & 208 & 189 & 999 & 1035 & 2718 & 2831 & 5198 & 5289 & 8272 & 8531 \\
\hline & 2 & 62.5 & 60.1 & 371 & 375 & 876 & 939 & 1792 & 1776 & 2877 & 2939 \\
\hline & 3 & 23.1 & 22.1 & 146 & 143 & 338 & 359 & 749 & 734 & 1208 & 1197 \\
\hline
\end{tabular}

${ }^{a}$ Monte Carlo simulation; b, $T_{k l} p^{-\beta_{k}}\left(\beta_{1}=0.56, \beta_{2}=0.75, \beta_{3}=0.72\right)$, where $T_{k l}$ is the relaxation times $T_{k}$ of linear polymer chains. 
Approximation Simulation of Dynamics of Lattice Model for Comb Polymer Chains

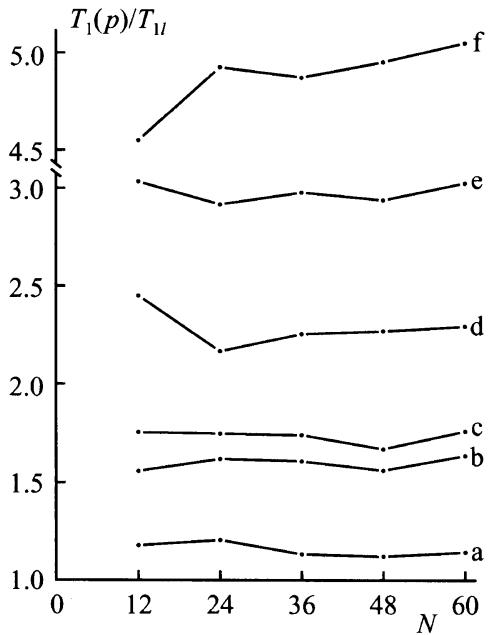

Figure 3. Plots of the quanitity $T_{1}(p) / T_{1 l} v s$. $N$ for a, $p=0.75 ; \mathrm{b}, p=0.5 ; \mathrm{c}, p=0.4 ; \mathrm{d}, p=0.3 ; \mathrm{e}, p=0.2 ; \mathrm{f}, p=0.1$ in the absence of excluded volume.

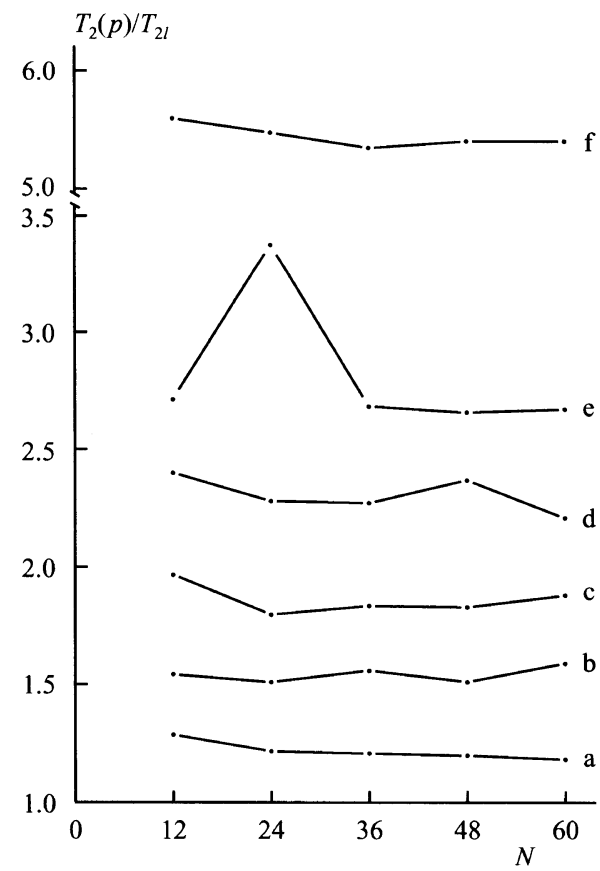

Figure 4. Plots of the quantity $T_{2}(p) / T_{2 l} v s . N$ in the absence of excluded volume. Symbols the same as in Figure 3.

The relaxation times of the first three normal modes, $T_{k}$, obtained from the slopes of the $\ln \rho_{k}(t) v s . t$ plots, are given in Tables II and III in the absence and presence of excluded

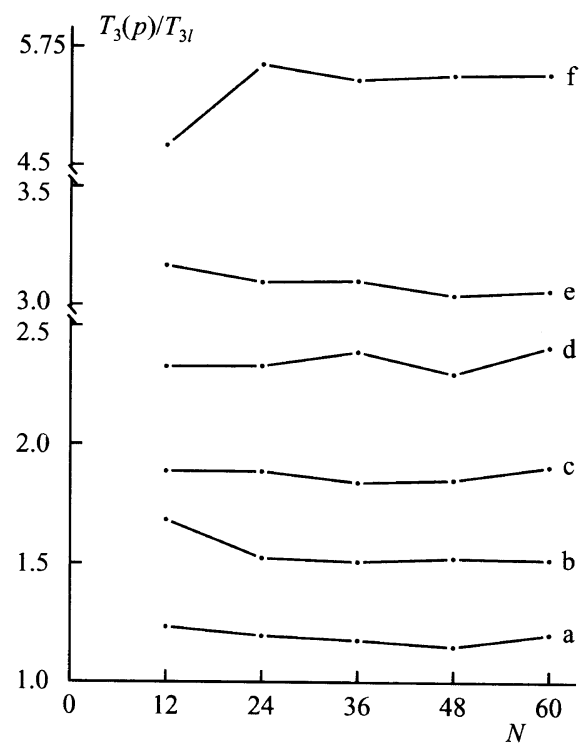

Figure 5. Plots of the quantity $T_{3}(p) / T_{3 l} v s . N$. Symbols the same as in Figure 3.

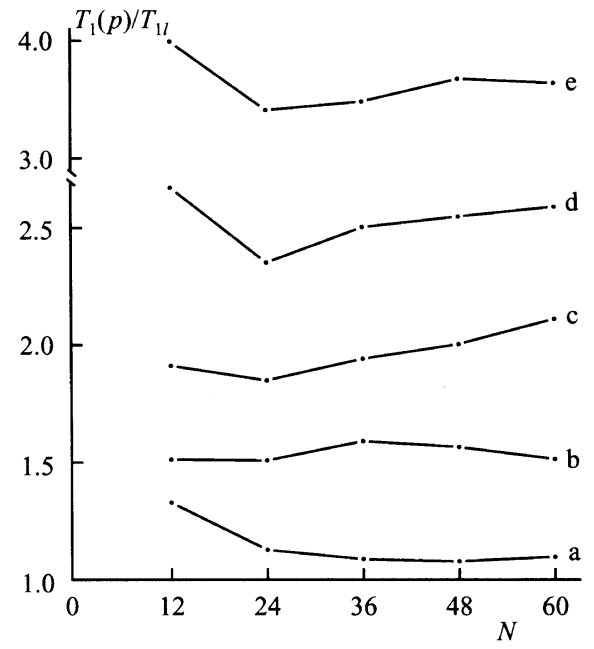

Figure 6. Plots of the quantity $T_{1}(p) / T_{1 l}$ vs. $N$ for a, $p=0.5 ; \mathrm{b}, p=0.4 ; \mathrm{c}, p=0.3 ; \mathrm{d}, p=0.2 ; \mathrm{e}, p=0.1$ in the presence of excluded volume.

volume, respectively. For a linear polymer chain, the relaxation times, $T_{k l}$, are given by

$$
\begin{aligned}
& T_{k l}-(N-1)^{\alpha_{k}} \\
& T_{k l}-k^{-\gamma_{N}}
\end{aligned}
$$

The values of $\alpha_{k}$ and $\gamma_{N}$ in the absence and 


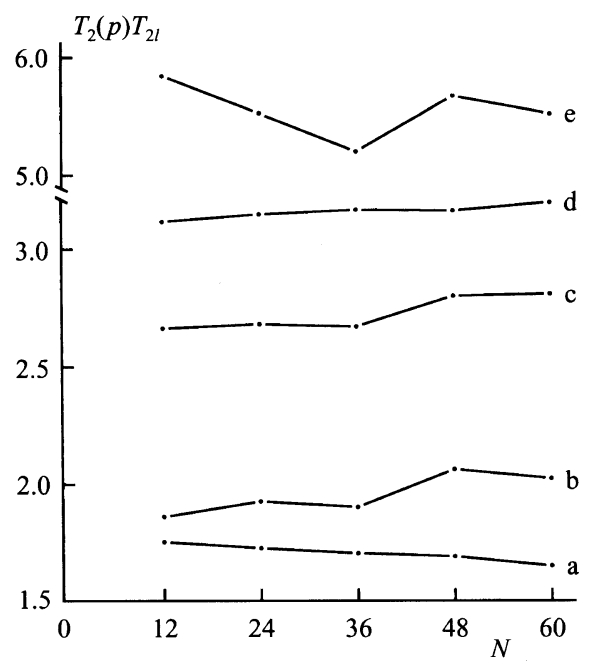

Figure 7. Plots of the quantity $T_{2}(p) / T_{2 l} v s . N$. Symbols the same as in Figure 6.

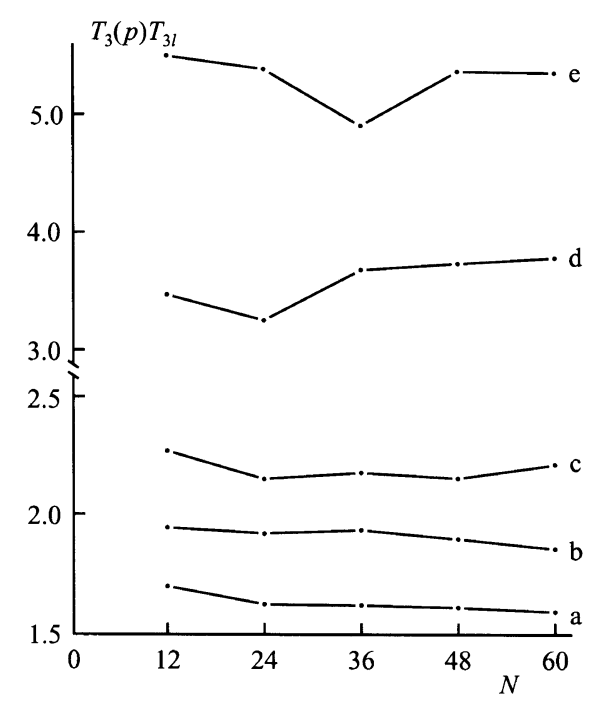

Figure 8. Plots of the quantity $T_{3}(p) / T_{3 l} v s$. $N$. Symbols the same as in Figure 6.

presence of excluded volume are 1.96, 1.97, and $2.17,2.17$, respectively, which are in good agreement with previous data. ${ }^{4,5}$ For the equifinal polymer chain, the relation between relaxation times $T_{K}$ and probability of movement $p$ is essentially the same as that of $T_{R}$. With increasing $p$, the chain moves fast, and relaxation times $T_{k}$ decrease. In order to investigate the effects of the side chain on relaxation times, we calculate $T_{k}(p) / T_{k l}$, and the results are given in Figures 3-8. In Figures 3 -5, we find $T_{k}(p) / T_{k l}$ in the absence of excluded volume to be nearly the same for various lengths of beads. The relation between relaxation times $T_{k}(p)$ and $p$ was investigated further, and it was found that the relaxation times, $T_{k}(p)$, obey the relation

$$
T_{k}(p)-(N-1)^{1.96} / k^{1.97} \cdot p^{0.70}
$$

The values of $T_{k}(p)$, calculated from eq 6 , are given in column $b$ of Table II, and the largest deviation from Monte Carlo simulation was only $10 \%$. In Figures $6-8$, the ratios $T_{k}(p) / T_{k l}$ in the presence of excluded volume are also the same for various lengths of beads, and the relaxation times are determined by

$$
T_{k}(p)-T_{k l} / p^{\beta_{k}}
$$

with $\beta_{1}=0.56, \beta_{2}=0.75, \beta_{3}=0.72$. The values of $T_{k}(p)$, calculated from eq 7 , are also given in column b of Table III, and the deviation from Monte Carlo simulation was no more than $17 \%$.

Here the dynamics of comb polymer chains are studied approximately. By investigating the configuration statistics of polymer chains with side chains, we find that the effects of the side chain $\mathrm{R}$ on the configuration statistics of polymer chain are insignificant. ${ }^{7,8}$ In this paper, the side chain $\mathrm{R}$ affects the dynamics of the polymer chains obviously, especially large side chains. Although the relations between relaxation times and probability of movement have been performed, we cannot study clearly the relation between the probability of movement and side chains. In the future some experiments will be made to clarify to what extent the approximation can be applied and assess the accuracy of the calculated results.

\section{REFERENCES}

1. P. H. Verdier and W. H. Stockmayer, J. Chem. Phys., 36, 227 (1962). 
Approximation Simulation of Dynamics of Lattice Model for Comb Polymer Chains

2. P. E. Rouse, J. Chem. Phys., 21, 1273 (1953).

3. F. Geny and L. Monnerie, J. Polym. Sci., Polym. Phys. Ed., 17, 131 (1979).

4. J. P. Downey and J. Kovac, Macromolecules, 20, 1357 (1987).

5. J. P. Downey, C. C. Crabb, and J. Kovac,
Macromolecules, 19, 2202 (1986).

6. L. Zhang, A. Xia, and J. Xu, Polym. J., 24, 1397 (1992).

7. L. Zhang and K. Gong, Eur. Polym. J., 29, 1631 (1993).

8. H. Ma and L. Zhang, Polym. J., 26, 121 (1994). 